\title{
Xantoastrocitoma pleomórfico espinal. Caso clínico
}

\author{
J.A. Simal-Julián; R Sanchis-Martín*; R. Prat-Acín; P. Miranda-Lloret; R. Conde-Sardón; E. Cárdenas-Ruiz- \\ Valdepeñas y A. Beltrán-Giner \\ Servicio de Neurocirugía Hospital La Fe de Valencia. España. Servicio de Anestesiología, Reanimación y Tratamiento del Dolor*. Hospital \\ General Universitario de Valencia. España.
}

Resumen

Introducción. Presentamos los aspectos clinicopatológicos y radiológicos de uno de los escasos casos de xantoastrocitoma pleomórfico espinal publicado, una entidad neoplásica infrecuente en una realmente rara localización. Revisamos la breve literatura inglesa disponible y establecemos un apropiado manejo en función de ésta.

Caso clínico. Mujer de 60 años de edad que consultó por acorchamiento progresivo de su mano izquierda, acompañado de ocasionales parestesias de dos meses de evolución. La exploración neurológica mostró debilidad y un leve trastorno de la sensibilidad propioceptiva de la extremidad superior derecha. El diagnóstico diferencial tras las pruebas de imagen se estableció entre astrocitoma y ependimoma. La paciente recibió tratamiento quirúrgico obteniéndose una resección completa y el diagnóstico de xantoastrocitoma pleomórfico. En los controles de imagen realizados a los $6,12,24$ y 36 meses no se objetivó recidiva tumoral. Actualmente la paciente ha recuperado su calidad de vida previa.

Discusión y conclusión. Comparando con los xantoastrocitomas pleomórficos intracraneales, aquellos con localización espinal (XAPE) presentan diferentes características epidemiológicas, con afectación predominante de niveles cervical y dorsal alto. La hipótesis de comportamiento más agresivo de los XAPE podría ser corroborada tras la revisión de la literatura. El estudio de extensión es fundamental para descartar la descrita diseminación a través del neuroeje. El grado de extensión de la resección quirúrgica es crucial en la prevención de la recurrencia tumoral. La radioterapia adyuvante debería únicamente considerarse cuando aparece tumor residual y/o anaplasia. Ensayos clínicos randomizados y bases de datos multicéntricas son necesarias para conocer todos los aspectos de esta entidad neoplásica.

Recibido: 16-02-10. Sceptado: 1-06-10
PALABRAS CLAVE: Espinal. Tratamiento. Xantoastrocitoma pleomórfico.

Spinal pleomorphic xantoastrocytoma. Case report

Summary

Introduction. We report the clinical, radiological and pathological features of a spinal pleomorphic xanthoastrocytoma, an unusual neoplastic entity in a really rare location, establish an appropriated management of these lesions and review the short available english literature.

Case report. A 60 years old woman consulted with doctor because she felt progressive clumsiness accompanied by occasional paresthesias on her left hand. Neurological examination showed up weakness and slight propioceptive disturbances. The differential imagine diagnosis was established between intramedullary astrocytoma and ependimoma. Patient underwent surgical gross total remove. Histopathological examination confirmed the diagnosis of pleomorfic xanthoastrocytoma. We performed MRI controls at 6, 12, 24 and 36 months that did not reveal recurrence. Nowadays, the patient has regained her previous quality of life.

Discussion and conclusion. Comparing to published cases about intracranial pleomorphic xanthoastrocytomas, spinal pleomorphic xanthoastrocytomas (SPXA) present different epidemiological characteristics. The known SPXAs affected to cervical and/or high thoracic levels. The hypothesis about a more aggressive behaviour of PXA in spinal cord may be corroborated after literature review. Extension examination is mandatory since dissemination along the neuroaxis has been described. Removal extension is crucial in the prevention of tumour recurrence. Adyuvant radiotherapy should only be considered when there is postoperative residual tumour and/or anaplastic features. Randomized clinical trials or databases are necessary to know all the aspects of this pathological entity. 
KEY WORDS: Pleomorphic xanthoastrocytoma. Spinal treatment.

\section{Introducción y objetivo}

El xantoastrocitoma pleomórfico (XAP) es una entidad neoplásica definida por Kepes y cols. en $1979^{8,11}$. Más tarde en 1993, fue incluida en la clasificación de tumores del sistema nervioso central de la $\mathrm{OMS}^{8,9,12,13}$. El XAP es asociado con un intervalo libre de recurrencia y supervivencia prolongados con una media de 18 años $^{19}$. A pesar del relativo buen pronóstico, el XAP puede recidivar y un $15-20 \%$ de dichos tumores recidivados podrían presentar transformación anaplásica ${ }^{2,3,18,22}$.

El XAP se presenta predominantemente a lo largo de las primeras cuatro décadas de vida, siendo más frecuente la localización supratentorial en el 98\% de los casos, involucrando el lóbulo temporal en el 50-60\% de los pacientes ${ }^{4,19}$. En la literatura inglesa disponible sólo hemos encontrado tres casos publicados con una localización espinal ${ }^{2,4,18}$.

Las características histológicas de los XAP son realmente típicas y comprenden pleomorfismo celular y nuclear, depósitos lipídicos citoplasmáticos, abundantes fibras reticulínicas estromales, inmunopositividad citoplasmática a la proteína ácida gliofibrilar, crecimiento tumoral en relación a las leptomeninges en la mayoría de los pacientes e implantación al menos parcial del plasmalema de las células tumorales a la lámina basal. Las mitosis son vistas ocasionalmente, siendo rara la necrosis, solamente hallada entorno al $11 \%^{1,13}$. Una de sus características más importantes de estas neoplasias es el comportamiento relativamente benigno a pesar del pleomorfismo histológico ${ }^{1,7,20}$.
En nuestro trabajo describimos los aspectos clinicopatológicos y radiológicos de uno de los escasos casos publicados en la literatura inglesa de xantoastrocitoma pleomórfico espinal (XAPE), una infrecuente localización para dicho tipo de tumores, revisamos los casos anteriormente documentados y establecemos su adecuado manejo basándonos en la escasa literatura disponible.

\section{Caso clínico}

Mujer de 60 años de edad que consultó por acorchamiento progresivo de su mano izquierda, acompañado de ocasionales parestesias de dos meses de evolución. La exploración neurológica mostró debilidad, leve alteración propioceptiva y reflejos osteotendinosos exaltados en el miembro superior derecho. No existió trastorno esfinteriano ni otros hallazgos.

El estudio mediante resonancia magnética reveló en las imágenes potenciadas en $\mathrm{T} 1$ una masa intramedular a nivel C4-C5 de 10x8x6 milímetros, anclada a la vertiente dorsal de la médula espinal, con intenso y homogéneo realce tras la administración de gadolinio. Asociada a esta lesión se objetivaba una cavidad siringomiélica desde región bulbar hasta T3 distalmente (Fig. 1). El estudio radiológico se completó con RM craneal y espinal sin hallazgos positivos. El diagnóstico diferencial se estableció entre astrocitoma y ependimoma.

Bajo anestesia general y en posición decúbito prono, la paciente es intervenida mediante un abordaje posterior con monitorización de potenciales evocados intraoperatorios. Se realizó laminectomía C4-C5, durotomía y mielotomía media posterior. Durante el procedimiento se evidenció un

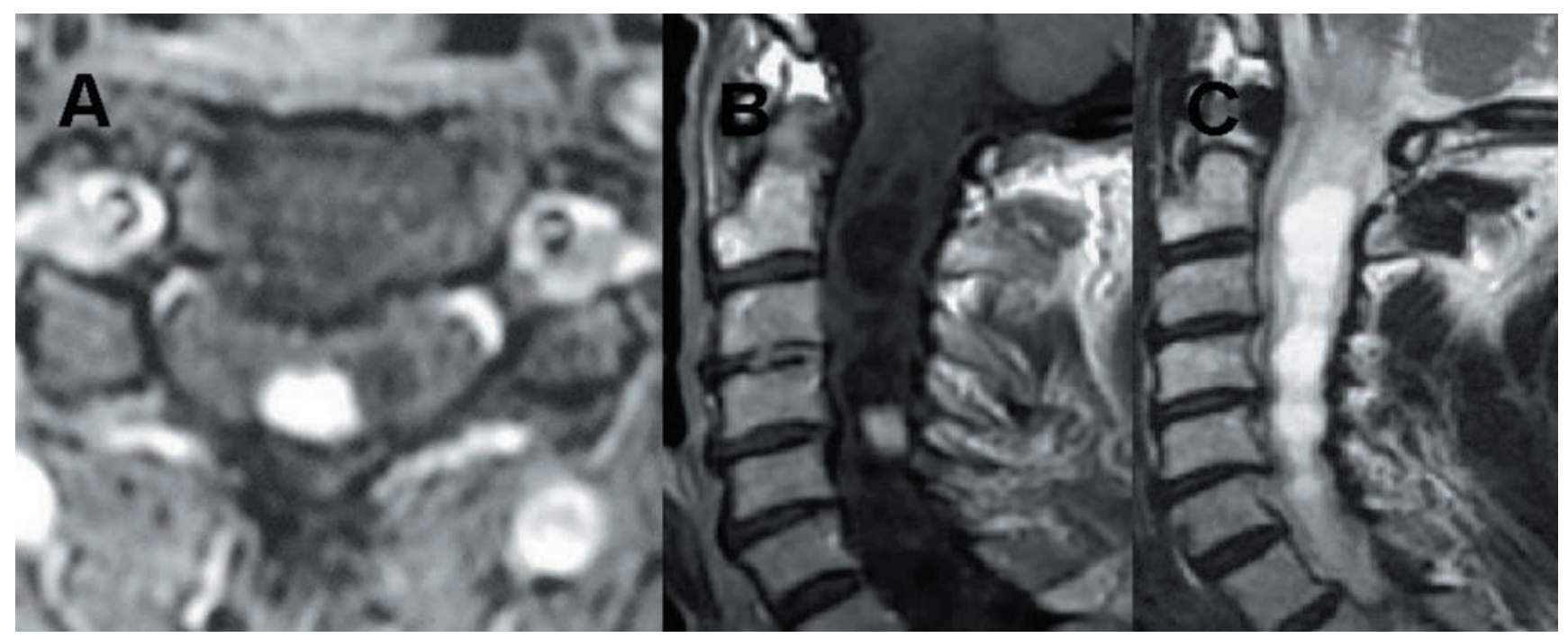

Figura 1. Imágenes preoperatorias de resonancia magnética potenciada en T1 con contraste axial (A) y sagital (B) revelan lesión intramedular de 10x8x6 millimetros a nivel C4-C5, con captación homogénea e intensa tras la administración de gadolinio. (C). Imagen RM potenciada en T2 muestra cavidad siringomiélica desde bulbo hasta D3. 


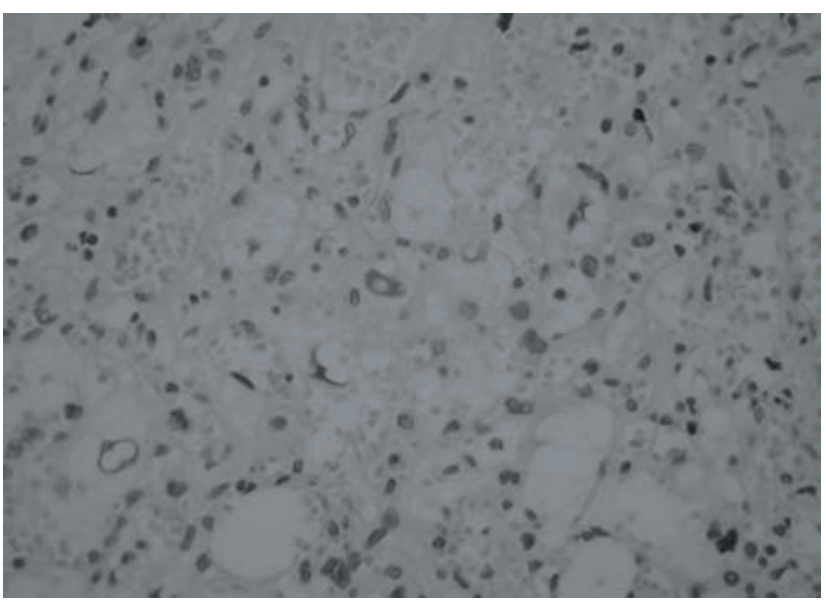

Figura 2. Tinción H\&E que demuestra tumor de características gliales con intenso pleomorfismo celular (citoplasmas vacuolados, núcleos hipercromáticos y grotescos).

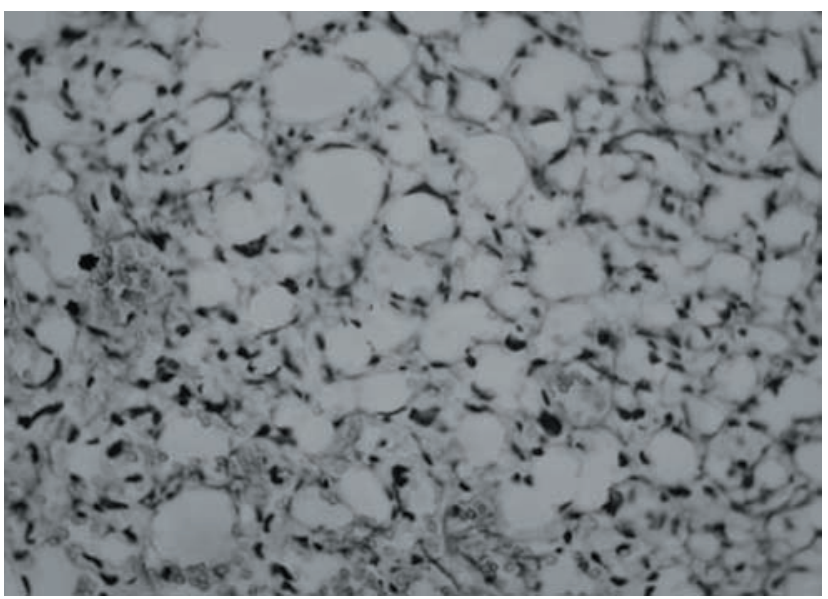

Figura 4. Positividad intensa para la proteina gliofibrilar ácida.

significativo anclaje del tumor a las leptomeninges y una transición xantocrómica que hizo posible una fácil disección y la resección total de la lesión.

El examen anatomopatológico confirmó el diagnóstico de XAP. Las tinciones con hematoxilina-eosina (Fig 2) pusieron de manifiesto tejido tumoral de características gliales, moderadamente celular, con células pleomórficas mono o binucleadas, citoplasmas vacuolados y núcleos hipercromáticos y grotescos. Una densa estructura reticulínica rodea pequeños grupos de células o células aisladas tal y como demuestra la tinción de Gomori (Fig 3). Se observó un índice mitótico bajo, menor del 1\% y ausencia de necrosis. Las células tumorales presentaron positividad para proteína gliofibrilar ácida (Fig .4) y proteína S100, mientras que fueron negativas para sinaptofisina, cromogranina y neurofilamentos.

La evolución postoperatoria fue tórpida e incluyó la presentación de meningitis bacteriana e hidrocefalia

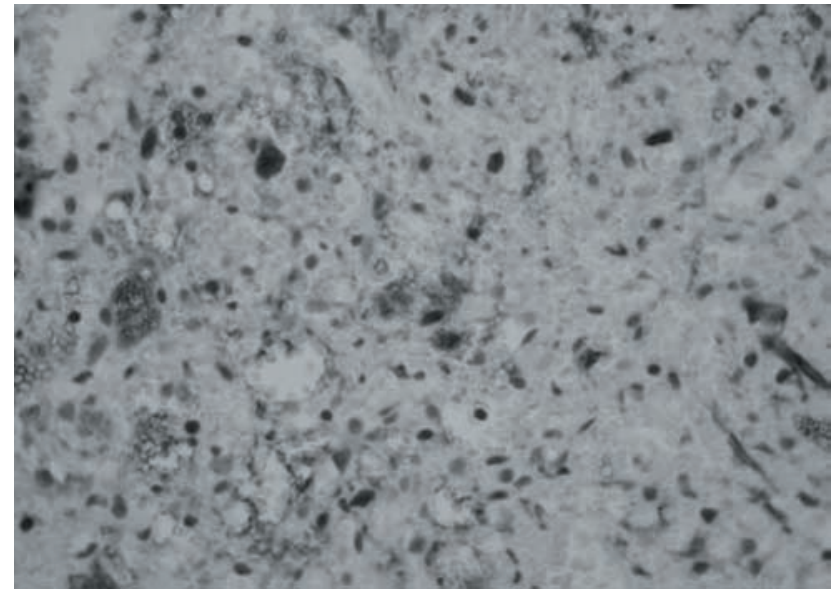

Figura 3. Tinción de Gomori que muestra denso entramado de fibras de reticulina que rodean grupos celulares.

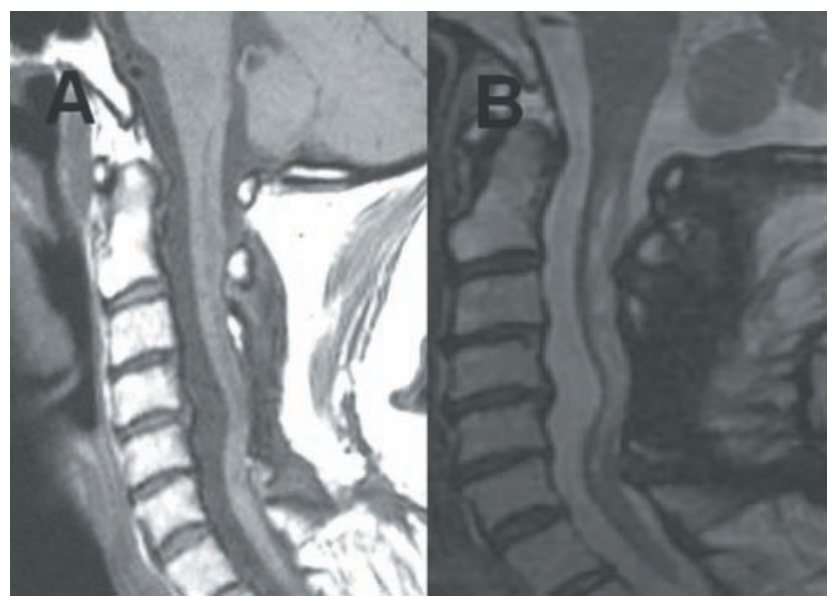

Figura 5. Imágenes postoperatorias de RM sagital potenciada en T1 tras la administración de contraste (A) y potenciada en $T 2$ (B) que revelan resección total del tumor, retracción dorsal del cordón medular y una significativa reducción de la cavidad siringomiélica.

secundaria que precisó la colocación de un sistema de derivación ventriculoperitoneal, proceso al que se solapó la concurrencia de un accidente isquémico transitorio. Finalmente la paciente pudo ser dada de alta presentando como única alteración un discreto trastorno de la sensibilidad propioceptiva en su extremidad superior derecha.

La paciente no recibió otros tratamientos complementarios. Se realizaron estudios de control mediante resonancia magnética a los 6, 12, 24 y 36 meses que no revelaron recidiva tumoral. El estudio realizado a los seis meses mostró una reducción significativa de la cavidad siringomiélica y una retracción de la médula espinal en el nivel intervenido (Fig 5). En la última revisión la paciente ha recuperado su estado funcional y la calidad de vida previa a la aparición de los síntomas que propiciaron el diagnóstico. 


\section{Discusión y conclusión}

Las características epidemiológicas de los casos de XAPEs publicados, en comparación con los xantoastrocitomas pleomórficos intracraneales (XAPIC) son diferentes. La edad en el momento del diagnóstico es mayor en las primeras, con una media de edad de 42 años, frente a la media de 26 años establecido para los XAPIC ${ }^{3}$. Respecto al sexo, los XAPEs afectaron predominantemente a mujeres, únicamente uno de los cuatro casos descritos, el más joven, fue varón, mientras que entre los XAPIC existe uniformidad respecto a la distribución de $\operatorname{sexos}^{3}$.

Respecto a la localización, los XAPEs conocidos afectaron a la médula cervical y/o dorsal alta. El primero de ellos, descrito por Herpers en $1993^{4}$, involucró los segmentos C5-D1, del segundo de ellos por orden de publicación no disponemos de datos ${ }^{2}$, el tercero afectó desde D2-D4 ${ }^{18} \mathrm{y}$ el descrito en este trabajo C4-C5.

El caso que recogemos presenta hallazgos intraoperatorios típicos de estos tumores, entre ellos la ya referida relación con las leptomeninges, supuestamente debido a un origen tumoral en los astrocitos subpiales, tal y como ponen de manifiesto los estudios mediante microscopia electrónica e inmunohistoquimia ${ }^{5,6,10,11,16}$.

Radiológicamente el diagnóstico diferencial es obligado plantearlo entre astrocitoma y ependimoma, lesiones intramedulares más frecuentes y habitualmente con componente quístico. Por su extremada rareza es difícil establecer el diagnóstico inicial de XAPE, la característica más típica de estas lesiones es la estrecha relación y el anclaje a las leptomeninges ${ }^{1}$.

Los casos de XAPEs descritos presentan cavidades siringomiélicas asociadas, sin captaciones patológicas de sus paredes. Por su parte, las lesiones de localización intracraneal van asociadas a una formación quística en un importante porcentaje de $\operatorname{casos}^{19}$, con un contenido xantocrómico en su interior con captación de contraste únicamente en el nódulo mural. En el caso de la localización espinal ninguno de los autores hace referencia al contenido de las cavidades siringomiélicas ni a su exploración quirúrgica, ni tampoco fue realizada por nuestra parte, pero dada la evolución postoperatoria nos inclinamos por interpretarlas como fenómenos de hidromielia.

Giannini y cols. ${ }^{3}$ obtuvieron una supervivencia total de $81 \%$ a los 5 años y $70 \%$ a los 10 , con una tasa de supervivencia libre de recurrencia de $72 \%$ a los 5 años y $61 \%$ a los 10 años tras analizar su serie de 71 XAPIC, mientras que únicamente dos de los cuatro XAPEs se encontraban vivos y libres de recurrencia a los 3 años del diagnóstico. Herpers y cols. ${ }^{4}$ sugirieron la posibilidad de un comportamiento más agresivo de los XAPEs, lo que podría parecer una conclusión precipitada ya que presentaron un caso aislado sin referentes previos en la literatura, pero los casos posteriormente descritos concuerdan con esta hipótesis.

Una de las diferencias fundamentales entre el nuestro y los casos restantes es el estudio de extensión que ninguno de los anteriores autores refieren en sus trabajos. Éstos obvian la posibilidad de diseminación tumoral a lo largo del neuroeje descrita tanto en casos de XAP anaplásicos como no anaplásicos ${ }^{14,21}$ y que podría justificar por otra parte el mal pronóstico referido anteriormente.

Existe una profunda controversia respecto al pronóstico de los XAP y la efectividad de la cirugía y otras terapias en su tratamiento. Macaula y cols. ${ }^{15}$ en su estudio sobre XAPIC establecieron en 1993 el mal pronóstico asociado a la presencia de características anaplásicas en el tumor

Tabla1

Casos publicados de xantoastrocitoma pleomórfico espinal

\begin{tabular}{|l|c|c|c|c|c|c|l|l|}
\hline \multicolumn{1}{|c|}{ Caso } & Sexo & Edad & $\begin{array}{c}\text { Clinic } \\
\text { Present }\end{array}$ & Loc. & Resec. & $\begin{array}{c}\text { Adyuv. } \\
\text { Radiot. }\end{array}$ & Recur. & Evoluc. \\
\hline $\begin{array}{l}\text { Herpers } \\
\mathbf{1 9 9 4}\end{array}$ & $\mathrm{M}$ & 66 & $\begin{array}{c}\text { Hipoestesia } \\
\text { T1-T12 }\end{array}$ & T2-T4 & Total & $\begin{array}{l}\text { Sí, tras } \\
\text { segunda } \\
\text { cirugía }\end{array}$ & $\begin{array}{l}\text { Sí. Local } \\
\text { tras 8 meses } \\
\text { postcirugía }\end{array}$ & $\begin{array}{l}\text { Hipoestesia } \\
\text { y paraparesia }\end{array}$ \\
\hline $\begin{array}{l}\text { Fouladi } \\
\mathbf{2 0 0 1}\end{array}$ & $\mathrm{H}$ & 12 & Cefalea & --- & Total & Sí & $\begin{array}{l}\text { Sí. Local y } \\
\text { metástasis }\end{array}$ & $\begin{array}{l}\text { Muerto por } \\
\text { enfermedad }\end{array}$ \\
\hline $\begin{array}{l}\text { Nakamura } \\
\mathbf{2 0 0 6}\end{array}$ & $\mathrm{M}$ & 33 & Hipoestesia & C5-T1 & Total & No & $\begin{array}{l}\text { No. 3 años } \\
\text { postcirugía }\end{array}$ & $\begin{array}{l}\text { Hipoestesia } \\
\text { nivel L1 }\end{array}$ \\
\hline $\begin{array}{l}\text { Actual } \\
\mathbf{2 0 1 0}\end{array}$ & $\mathrm{M}$ & 60 & Paresia & C4-C5 & Total & No & $\begin{array}{l}\text { No. 3 años } \\
\text { postcirugía }\end{array}$ & $\begin{array}{l}\text { Trastorno } \\
\text { propioceptivo }\end{array}$ \\
\hline
\end{tabular}


primario o de la recurrencia en el recidivado, con supervivencias menores de un año en la mayoría de los casos evaluados. Definieron anaplasia como una alta actividad mitótica, marcada celularidad, hipercromatismo nuclear, hiperplasia endotelial, necrosis geográfica y disposición nuclear en empalizada. El grado de resección total o parcial, o el tratamiento con radioterapia no se asociaron a diferencias en la supervivencia. Aunque su revisión no fue capaz de identificar factores pronósticos, estos autores sugirieron un posible beneficio de la resección quirúrgica completa. Más tarde, en 1996, Pahapill y cols. ${ }^{19}$ analizaron 79 pacientes identificados en la literatura inglesa desde 1979 hasta 1994 con XAP de localización intracraneal. Este estudio mostró que la inclusión de pacientes con XAP y que habían presentado necrosis histológica en el estudio de Macaula y cols. ${ }^{15}$ había sesgado sus conclusiones, ya que si los pacientes con necrosis eran excluidos, la resección completa sería claramente beneficiosa. Por el contrario, la resección completa no supondría grandes beneficios para los pacientes con tumores que presentan necrosis. Respecto al papel de la radioterapia, sus análisis no encontraron influencia en el pronóstico de estos tumores, pero sugirieron el posible papel beneficioso de la radioterapia adyuvante en los casos de XAP sin necrosis. Giannini y cols. ${ }^{3}$ en 1999 revisaron 71 pacientes con lesiones intracraneales y realizaron un análisis uni y multivariante concluyendo que la extensión de la resección y el índice mitótico eran predictores independientes de recurrencia y supervivencia libre de enfermedad y que solamente el índice mitótico era factor independiente para la supervivencia. La presencia de necrosis no tuvo significación, así que plantearon designar XAP anaplásico basándose en su índice de proliferación con o sin necrosis acompañante. El papel de la radioterapia y quimioterapia adyuvantes no fue claramente establecido por estos autores.

Aunque las series de XAP espinales e intracraneales no son comparables, dado lo limitado del número de casos de los primeros y la dificultad para extraer conclusiones y establecer pautas de actuación, consideramos fundamental el conocimiento y descripción de las series de localización intracraneal descritas en párrafos anteriores.

En el caso presentado, se alcanzó una resección total y no se aplicaron tratamientos complementarios, tal y como ocurrió con el caso expuesto por Nakamura y cols ${ }^{18}$, dado que el estudio anatomopatológico no mostró ni necrosis ni alto índice mitótico. Herpers y cols. ${ }^{4}$ tampoco hallaron en su caso signos de anaplasia, pero el corto tiempo libre de recurrencia, 8 meses, y la resección parcial conseguida ${ }^{8,19}$ justificaron la administración de radioterapia adyuvante tras la segunda cirugía.

El grado de resección es crucial en la prevención de recurrencia tumoral. Tanto en nuestra experiencia como en la disponible en la literatura consultada, la radioterapia adyuvante únicamente debería ser considerada cuando exista tumor residual y/o características anaplásicas. Se deberían realizar no obstante, ensayos clínicos randomizados y bases de datos multicéntricas para poder conocer mejor todos los aspectos de esta entidad neoplásica.

\section{Bibliografía}

1. Allegranza, A., Farraresi, S., Bruzzone, M., Giombini, S.: Cerebromeningeal pleomorphic xanthoastrocytoma: Reporto on tour cases: Clinical, radiologic and pathologic features (Including a case with malignant evolution). Neurosurg Rev 1991; 14: 43-49.

2. Fouladi, M., Jenkins, J., Burger, P., Langston, J., Merchant, T., Hideman, R., Thomson, S., Sandford, A., Kun, L., Gajjar: A pleomorphic xanthoastrocytoma: favourable outcome after complete surgical resection. Neurooncology 2001; 3: 184-192.

3. Giannini, C., Scheithauer, B.W., Burger, P.C., Prat, D.J., Wollan, P.C., Lach, B., et al: Pleomorphic xanthoastrocytoma: what do we really know about it? Cancer 1999; 85: 20332045.

4. Herpers, M.J., Freling, G., Beuls, E.A.: Pleomorphic xanthoastrocytoma in the spinal cord: Case report. J Neurosurg 1994; 80: 564-569.

5. Heyerdahl Strom, E., Skullerud, K.: Pleomorphic xantoastrocytoma: report of 5 cases. Clin Neuropathol 1983; 2 : 188-191.

6. Iwaki, T., Fukui, M., Kondo, A., et al.: Epithelial properties of pleomorphic xanthoastrocytomas determined in ultrastructural and immunohistochemical studies. Acta Neuropathol 1987; 74: 142-150.

7. Kaulich, K., Blaschke, B., Numann, A., von Deimling, A., Wiestler, O.D., Weber, R.G., Reifenberger, G.: Genetic alterations commonly found in diffusely infiltrating cerebral gliomas are rare or absent in pleomorphic xanthoastrocytomas. J Neuropathol Exp Neurol 2002; 61: 1092-1099.

8. Kepes, J.J.: Pleomorphic xanthoastrocytoma: The birth of a diagnosis and a concept. Brain Pathol 1993; 3: 269-274.

9. Kepes, J.J., Kepes, M., Slowik, F.: Fibrous xanthomas and xanthosarcomas of the meninges and the brain. Acta Neuropathol 1973; 23: 187-99.

10. Kepes, J.J., Rubinstein, L.J., Ansbacher, L., Schreiber, D.J.: Histopathological features of recurrent pleomorphic xanthoastrocytomas: Further corroboration of the glial nature of this neoplasm-A study of three cases. Acta Neuropathol (Berl) 1989; 78: 585-593.

11. Kepes, J.J., Rubinstein, L.J., Eng, L.F.: Pleomorphic xanthoastrocytoma: A distinctive meningocerebral glioma of young subjects with relatively favorable prognosis-A study of 12 cases. Cancer 1979; 44: 1839-1852.

12. Kleihues, P., Burger, P.C., Scheithauer, B.W.: Astrocytic Tumours, in Histological Typing of Tumours of the Central 
Nervous System, ed 2. Berlin: Springer-Verlag, 1993, pp1115.

13. Lopes, M.B.S., VandenBerg, S.R., Scheithauer, B.W.: The World Health Organization classification of nervous system tumors in experimental neurooncology. In: Levine AJ, Schmidek HH (eds) Molecular genetics of nervous system tumors, Wiley-Liss, New York, 1993, pp 1-36.

14. Lubansu, A., Rorive, S., David, P., Sariban, E., Selingmann, R., Brotchi, J., Pirotte, B.: Cerebral anaplastic pleomorphic xanthoastrocytoma with meningeal dissemination at first presentation. Childs Nerv Syst 2004; 20: 119-122.

15. Macaulay, R.J., Jay, V., Hoffman, H.J., Becker, L.E.: Increased mitotic activity as a negative prognostic indicator in pleomorphic xanthoastrocytoma. J Neurosurg 1993; 79: 761767.

16. Maleki, M., Robitaille, Y., Bertrand, G.: Atypical xanthoastrocytoma presenting as a meningioma. Surg Neurol 1983; 20: 235-238.

17. Nakajima, T., Kumabe, T., Shamoto, H., Watanabe, M., Suzuki, H., Tominaga, T.: Malignant transformation of pleomorphic xanthoastrocytoma. Acta Neurochir (Wien) 2006; 148: 67-71.

18. Nakamura, M., Chiba, K., Matsumoto, M., Ikeda, E.,
Toyama, Y.: Pleomorphic xanthoastrocytoma of spinal cord: case report. J Neurosurg Spine 2006; 5: 72-75.

19. Pahapill, P.A., Ramsay, D.A., Del Maestro, R.F.: Pleomorphic xanthoastrocytoma: case report and analysis of the literature concerning the efficacy of resection and the significance of necrosis. Neurosurgery 1996; 38: 822-829.

20. Palma, L., Maleci, A., Di Lorenzo, N., Lauro, G.M.: Pleomorphic xanthoastrocytoma with 18-year survival. Case report. J Neurosurg 1985; 63: 808-810.

21. Passone, E., Pizzolitto, S., D’Agostini, S., Skrap, M., Gardiman, M.P., Nocerino, A., Scarzello, G., Perilongo, G.: Non-anaplastic pleomorphic xanthoastrocytoma with neuroradiological evidences of leptomeningeal dissemination. Childs Nerv Syst 2006; 22: 614-618.

22. Prayson, R.A., Morris, H.H.: Anaplastic pleomorphic xanthoastrocytoma. Arch Pathol Lab Med 1998; 122: 10821086.

Simal-Julián, J.A.; Sanchis-Martín, R.; Prat-Acín R.; Miranda-Lloret, P; Conde-Sardón, R.; Cárdenas-RuizValdepeñas, E; Beltrán-Giner, A.: Xantoastrocitoma pleomórfico espinal. Caso clínico. Neurocirugía 2010; 21: 390395. 